involvement and possible surgery. An early sign of Coats disease is a yellow-eye in flash photography, a reflection off cholesterol deposits in retinal blood vessels.

Coats disease is named after George Coats (Coats G. Forms of retinal disease with massive exudation. Royal London Ophthalmic Hospital Reports. 1908;17(3):440-525). A syndrome characterized by retinal, hearing, muscle and mental disorders was described 60 years later by Robert G. Small (Small RG. Coats' disease and muscular dystrophy. Transactions of the American Academy of Ophthalmology and Oto-Laryngology, Rochester, MN. 1968 Mar-Apr;72(2):225-31).

\title{
SMA TYPE III MIMICS MUSCULAR DYSTROPHY
}

Researchers at the National Neuroscience Institute, Riyadh, Saudi Arabia, report a series of 8 patients with type III spinal muscular atrophy who were referred with a diagnosis of muscular dystrophy. Developmental milestones were normal until early juvenile or teens years when they showed a slowly progressive proximal weakness involving limb-girdle muscles. A clumsy gait was associated with frequent falls and difficulty in climbing stairs. Seven patients were products of consanguineous marriage. Hypertrophy of calves in 3 patients contrasted with generalized muscle wasting. Tongue fasciculation occurred in 2 patients, deep tendon reflexes were diminished in 7 , and spinal scoliosis developed in 5. Muscle biopsy had nonspecific myopathic features in 3 patients, and nerve conduction studies showed normal, mildly neurogenic or myopathic changes. Serum creatine kinase levels varied from normal to significantly elevated. The diagnosis of SMA III was confirmed by gene testing where deletions of exon 7 were detected in all patients. (Alsaman AS, AlShaikh NM. Type III spinal muscular atrophy mimicking muscular dystrophies. Pediatr Neurol 2013 May;48(5):363-6). (Response: Dr Alsaman. E-mail: aalsaman@kfmc.med.sa).

COMMENT. In the diagnosis of SMA type III, the presence of dystrophic features such as calf muscle hypertrophy, limb-girdle muscle weakness, elevated serum $\mathrm{CPK}$, and myopathic or dystrophic muscle biopsy findings will sometimes lead to confusion with muscular dystrophy. Diagnosis is confirmed with a molecular genetic polymerase chain reaction-based test for $5 \mathrm{q}$ telomeric SMN1 mutation.

\section{VASCULAR DISORDERS}

\section{INTRACEREBRAL HEMORRHAGE, ACUTE SYMPTOMATIC SEIZURES, AND EPILEPSY}

Investigators at Yale University School of Medicine, New Haven, CT; Children's Hospital of Philadelphia; Vanderbilt University, Nashville, TN; and Johns Hopkins University, studied the incidence and risk factors for seizures and epilepsy in 73 children with spontaneous intracerebral hemorrhage $(\mathrm{ICH})$ including 20 perinatal subjects $(>37$ weeks gestation to 28 days) and 53 aged $>28$ days to $<18$ years at presentation. Acute symptomatic seizures occurred in 35 subjects $(48 \%)$; they were a presenting symptom of ICH in 12 perinatal (60\%) and 19 childhood (36\%) subjects, and they occurred after 
presentation in 7. Electroencephalographic-only seizures occurred in 9 of 32 subjects (28\%) with continuous EEG monitoring. One-year and 2-year remote symptomatic seizure-free survival rates were $82 \%$ and $67 \%$, respectively. One-year and two-year epilepsy-free survival rates were $96 \%$ and $87 \%$, respectively. Elevated intracranial pressure requiring acute intervention was a risk factor for seizures after presentation $(\mathrm{P}=0.01)$, for remote symptomatic seizures $(\mathrm{P}=0.03)$, and epilepsy $(\mathrm{P}=0.04)$. Single remote symptomatic seizures occur in many, and epilepsy is estimated to develop in $13 \%$ patients at 2 years after ICH presentation. (Beslow LA, Abend NS, Gindville MC, et al. Pediatric intracerebral hemorrhage. Acute symptomatic seizures and epilepsy. JAMA Neurol 2013 Apr 1;70(4):448-54). (Respond: Beslow LA, Section of Child Neurology, Yale University, New Haven CT. E-mail: lauren.beslow@yale.edu).

COMMENT. Acute symptomatic seizures were defined as those occurring up to 7 days after the incident $\mathrm{ICH}$, and remote symptomatic seizures as occurring beyond 7 days from ICH presentation. An editorial points out that acute symptomatic seizures with ICH occur more frequently in children (60\%) than in adult reports (7\%-31\%) (Heyer GL, Roach ES. JAMA Neurology 2013 Apr 1;70(4):437).

Hemisphere specific motor control mechanisms in post-stroke rehabilitation.

Left hemisphere damage is associated with greater errors in movement direction of the contralateral limb while errors in movement extent are greatest after right hemisphere damage. The differential deficits induced by right or left hemisphere lesions must be considered during post-stroke rehabilitation. (Mani S et al. Brain 2013 Apr;136(Pt 4):1288-303).

\section{RISK FACTORS FOR ARACHNOID CYST HEMORRHAGE}

Neurosurgeons at Primary Children's Medical Center, University of Utah, Salt Lake City, evaluated factors that are associated with rupture of arachnoid cysts (intracystic hemorrhage, subdural hematoma, or adjacent subdural hygroma) in children with previously asymptomatic arachnoid cysts. Two unruptured nonhemorrhagic controls were matched to each case. A total of 309 patients with treated and untreated arachnoid cysts were identified between 2005 and 2010, an institutional prevalence of 1.9\%. After exclusion of surgical cases, 232 remained in the study. Risk factors evaluated included arachnoid cyst size, recent head trauma, and altitude at residence. Fourteen cases $(6 \%)$ presented with either rupture or hemorrhage. Larger cyst size and diameter was significantly associated with cyst rupture/hemorrhage $(p<0.001)$. Recent history of trauma was also associated with outcome $(\mathrm{p}<0.01)$. Altitude was not a risk factor. Children with rupture/hemorrhage were more likely to present with headache or signs or symptoms of raised intracranial pressure, including midline shift. None suffered neurological sequelae. (Cress M, Kestle JRW, Holubkov R, Riva-Chambrin J. Risk factors for pediatric arachnoid cyst rupture/hemorrhage: A case-control study. Neurosurgery 2013 May;72(5):716-22). (Response: Jay Riva-Chambrin MD. E-mail: Jay.Riva-Cambrin@hsc.utah.edu).

COMMENT. Cyst size and recent head trauma are risk factors for cyst rupture. 